\section{Exotic small mammal pain management}

\section{Stephen Cital}

Pain management in these species is similar to that in the cat and dog with the exception of handling and restraint as well as dosing. Dosing ranges vary from text to text with ever-changing research into efficacy of drugs and dosages thus far described. It is imperative for the reader to be constantly researching the newest most supported methods in pain management for whatever species one is working with. Sites for analgesia delivery vary from species to species and feasibility depending on muscle mass for intramuscular injections, vascular access for intravenous injections and availability of subcutaneous space. The recent publications of the mouse, rabbit and rat facial pain-scoring charts are a crucial addition to any exotic practitioner's library.

As mentioned, the principal standards associated to cat and dog pain management apply to small exotic mammals in regard to first-line agents. Opioids are still a first-line option for acute or very painful animals. Nonsteroidal anti-inflammatory drugs are also good for acute and chronic pain states, while local anaesthetics are useful whenever practical and feasible. For exotics we can also consider alternative approaches, such as laser, herbal supplements, exercise and even acupuncture.

\section{KEY LEARNING OBJECTIVES}

- Current literature review of species dosing strategy

- Understand practical approaches to multimodal pain management in exotics

- Understand best practices for exotic mammals and species-specific concerns with pain management

\section{MULTIPLE CHOICE QUESTIONS}

1. What is considered one of the more versatile and effective opioid drugs for exotic mammals?
(A) Morphine
(C) Butorphanol
(B) Buprenorphine
(D) Nalbuphine

2. Local anaesthetics can be used safely in what species?
(A) Rabbits
(C) Rats
(B) Hedgehogs
(D) All of the above

3. Which of the following statements about the nociceptive system in exotic mammals is true?

(A) Just as robust and complex as in cats and dogs

(B) Almost as robust and complex as in cats and dogs

(C) Not as robust and complex as in cats and dogs

(D) Present but they do not perceive pain the same way

\title{
Local and regional nerve blocks
}

\section{Stephen Cital}

Local and regional anaesthesia (L\&RA) is the technique of applying or infiltrating tissues with a sodium-channel blocking agent (most common in veterinary medicine: lidocaine, bupivacaine, ropivicaine) to completely numb a specific area. We can literally take an animal in severe pain, such as broken ribs, and make them comfortable again within minutes. There are several adverse effects of continued pain, many of which delay healing and impact the patient psychologically. Local and regional blocking techniques are one of the few techniques we have to completely stop pain signaling to the spinal cord, further reducing sensitization, which in the worst-case scenario could lead to neuropathic pain. Local and regional blocking used for the anaesthetized patient also shows a decrease in morbidity and mortality and decreases complication in the postoperative period. There are multiple terms used for differing techniques of L\&RA.

Topical or surface anaesthesia is using sodiumchannel blocking agents in creams or solutions on the skin or mucous membrane providing some relief. Unfortunately, many of the agents we use are not readily

absorbed through the skin surface unless left on for quite some time. The use of lidocaine patches over wounds or incisions has also been described but has not been found to alleviate the need for other analgesic medications.

Local infiltration is a less precise means of infiltrating tissue with a blocking agent to achieve pain sensation loss. Basically, the local blocking agent should be used where the surgeon plans to incise or where tissues will be manipulated in a way that causes discomfort. There are many studies on the efficacy of using this technique for any stable surgical patient. The efficacy of this technique has even found its way into the Pain Management Guidelines published by the American Animal Hospital Association (AAHA).

Regional or nerve blocking techniques are a bit more precise using anatomical landmarks, palpation or devices to infiltrate the blocking solution within millimetres of a nerve. A good knowledge of the nervous system anatomy is desirable before implementing such techniques. It is important we are not piercing the actual nerve or infiltrating the nerve, like commonly done during leg amputations. More recent research has shown that infiltration of the nerve, stretching the fibres, can sensitize the remaining nerve component adversely.

Neuraxial anaesthesia is the technique of infiltrating blocking agents in the epidural space. This is a very effective technique for essentially anything on the caudal half of the animal and can be useful for such conditions as 